\title{
VICTIMIZACIÓN E IMPUNIDAD EN EL ESTADO DE MORELOS, MÉXICO ${ }^{1}$
}

\section{VICTIMIZATION AND IMPUNITY IN MORELOS STATE, MEXICO}

\author{
Elián Gómez-Azcárate* \\ J. Alejandro Vera** \\ María Elena Ávila* \\ Guadalupe Arias* \\ Enrique Vega \\ M. Joel $\operatorname{Arcos}^{* * * * * * *}$
}

\section{RESUMEN}

En este artículo se contrastó un modelo explicativo de la percepción de inseguridad, a través del análisis del papel de la impunidad percibida, programas de seguridad pública, confianza en autoridades y medidas preventivas, en la relación entre la victimización y la

1 Este estudio se ha elaborado en el marco del Proyecto de Investigación: Diagnóstico Estatal de la percepción ciudadana sobre la violencia, la delincuencia, la inseguridad, y la dinámica social e institucional. Proyecto financiado por el Fondo Mixto de Fomento a la Investigación Científica y Tecnológica. Consejo Nacional de Ciencia y Tecnología (CONACYT)-Gobierno del Estado de Morelos. Clave: MOR-2012-C01-190638. Se agradece a conAcYT por el financiamiento de la estancia Posdoctoral para la consolidación de grupos de Investigación \#000000000234767, asî como a los encuestadores y al equipo de investigación que hicieron posible este trabajo.

* $\quad$ Facultad de Comunicación Humana, Universidad Autónoma del Estado de Morelos, México. elian.gomez@uaem.mx

** Centro de Investigación Transdisciplinar en Psicología, Universidad Autónoma del Estado de Morelos, México. moises.arcos@uaem.mx

*** Centro de Investigación Transdisciplinar en Psicología, Universidad Autónoma del Estado de Morelos, México. meavila@uaem.mx

**** Investigador pensionado, Universidad Autónoma del Estado de Morelos, México. evegavi@gmail.com

****** Maestría en Psicología, Universidad Autónoma del Estado de Morelos, México. gualar@gmail.com

******* Cursando el Doctorado en Psicología, Universidad Autónoma del Estado de Morelos, México. moises.arcos@uaem.mx 
inseguridad. Participaron 8170 habitantes del Estado de Morelos, México. Los resultados mostraron que la victimización ejerció un efecto directo sobre impunidad y estas a su vez incrementaron la inseguridad percibida. Mientras que la confianza en las autoridades y los programas preventivos, disminuyeron la inseguridad a pesar del alto nivel de victimización. Los resultados tienen importantes implicaciones para el tema de la seguridad ciudadana.

PALABRAS CLAVE: MÉXICO * INSEGURIDAD * PREVENCIÓN DEL CRIMEN * PERCEPCIÓN * VIOLENCIA

\section{ABSTRACT}

In this article explicative model of the perception of insecurity was contrasted, through the analysis of the role played by impunity, prevention programs' expectation, trust on the authorities and prevention strategies in the relation between victimization and insecurity. The participants were 8170 inhabitants of Morelos State, Mexico. The results showed that victimization had a direct effect on impunity, which increased the perception of insecurity. While trust on the authorities and the prevention programs reduced the insecurity regardless of the victimization level. Results have important implications for the citizen's security.

KEYWORDS: MEXICO * INSECURITY * CRIME PREVENTION * PERCEPTION * VIOLENCE

\section{INTRODUCCIÓN}

La seguridad es uno de los pilares del buen gobierno $y$ es la base para el desarrollo pleno y equitativo de las personas (Carbonell, Orozco y Vázquez 2002, pnud 2013, Programa de las Naciones Unidas para los Asentamientos Humanos onu-habitat 2009). Sin embargo, en las últimas décadas en varios países latinoamericanos, entre ellos México, la seguridad se ha visto terriblemente deteriorada por el elevado nivel de violencia $y$ delincuencia, volviéndose el principal reto social (Rico y Chinchilla 2002, Programa de las Naciones Unidas para el Desarrollo-Pnud 2013). El estudio La violencia en los municipios y las entidades federativas de México, presentado por el Consejo Ciudadano para la Seguridad Pública y la Justicia Penal AC (2013), reveló que Morelos fue la segunda entidad federativa con el más elevado índice de violencia y delincuencia. En el 2014, según la Encuesta Nacional de Victimización y Percepción Sobre Seguridad Pública (envipe), en Morelos se reportaron 36524 delitos en total por cada 100 mil habitantes. Entre los delitos más frecuentes 11712 casos de robo o asalto en la calle o en el transporte público; 7299 casos de extorsión; 4318 casos de amenazas verbales, por cada 100 mil habitantes (Instituto Nacional de
Estadística y Geografía-INEGI 2014). Con una población aproximada de 1777 mil habitantes, se infiere un aproximado de 620 mil delitos en un año, que equivale a 1700 delitos al día, haciendo de esta entidad un contexto deplorablemente violento, en el cual la percepción colectiva de inseguridad es inevitable.

En México, la inseguridad percibida ha sido un tema tan relevante, como la delincuencia misma. Prueba de ello son las mediciones periódicas que ha realizado el gobierno a través del INEGI, desde el 2005, con la Encuesta Nacional Sobre Inseguridad (ENSI) y en los últimos años con la ENviPe (INEGI 2011, 2012, 2013 y 2014). Los últimos resultados muestran que a nivel nacional, la inseguridad fue la principal preocupación de casi seis de cada diez personas; en Morelos, fue apenas un poco menor $(5,8 / 10)$. En cuanto a la percepción de inseguridad, aproximadamente el 73,3\% de la población a nivel nacional, percibió inseguridad en su entidad federativa; mientras que en Morelos fue el 89\% de la población (INEgi 2014).

La percepción de inseguridad supone una sensación subjetiva de temor generada por la violencia directa o indirecta (Bonastra, Arella, Rodríguez y Fraile 2006, PNud 2013). Esto sugiere que no se tiene que ser víctima directa de un acto delictivo para percibir la pérdida de la 
seguridad. La percepción de inseguridad se basa en el sentido común de cada individuo, deriva de representaciones construidas por las colectividades (Reyes 2007) y puede llegar a afectar todos los ámbitos de la vida, tanto individual como de la comunidad, impidiendo el desarrollo humano (pNud 2013).

Dentro del estudio científico de la percepción de inseguridad, se ha visto una fuerte relación entre la victimización y la inseguridad percibida (Bailey y Flores-Macías 2007, Braakmann 2012, Dammert 2012). Se ha considerado que dicha relación, se explica en gran medida por el miedo a la revictimización criminal (Amerio y Roccato 2005). No obstante, la relación entre el riesgo subjetivo de convertirse en víctima y las tasas reales de victimización difieren en la mayoría de estudios, tanto en México (INEGi 2011, 2012, 2013 y 2014), como en otros países (Chadee y NgYing 2013, Hipp 2010, Zimring y Hawkins 1997); siendo en todos los casos, mucho más alta la sensación de riesgo y temor a ser víctima del delito, en comparación con los niveles reportados de victimización y de revictimización criminal. En este sentido, es importante considerar la victimización indirecta como otro factor asociado a la inseguridad. Las víctimas indirectas no son solo quienes sufren las consecuencias de la victimización de un familiar, amigo o alguna persona cercana, son también personas con miedo por el alto riesgo de ser víctima en contextos con altos niveles de violencia, delincuencia, etc. (onu 1985), como es el caso de los habitantes de Morelos, el segundo Estado más violento del país.

La percepción de inseguridad también se ha asociado con la falta de confianza en las instituciones públicas, especialmente en las de seguridad pública (Dammert 2010; Hernández 2008 y Ruíz 2007). Esta, conjuntamente con la insatisfactoria respuesta gubernamental ante el incremento de actos delictivos, acrecientan notoriamente la preocupación ciudadana, haciéndoles sentir más vulnerables ante un posible daño a sus bienes $y$ a su integridad física individual, reportando niveles cada vez más altos de inseguridad (Acero 2005). Al mismo tiempo, en la perspectiva de la ciudadanía mexicana, frecuentemente los canales institucionales pensados para atender las demandas sociales se encuentran inmóviles (Hernández 2008). En este sentido, la falta de interés de las autoridades o su inadecuada respuesta, puede incluso reforzar la idea de que las autoridades son culpables, en alguna forma, del nivel de delincuencia (onu 1985).

La desconfianza de la ciudadanía en las autoridades e instituciones gubernamentales se incrementa por la impunidad (Mier Garza 2010, PNuD 2013) y la corrupción percibidas (Jusidman 2010). En México, se advierte como la gente desconfía y/o duda de las instituciones encargadas de prevenir $y$ de contener la violencia $y$ la delincuencia; lo que a su vez se relaciona con el bajo nivel de denuncia de delitos por parte de la ciudadanía. A nivel nacional, en el 2013, aproximadamente el 90\% de los delitos no fueron denunciados. En Morelos, el porcentaje de delitos no denunciados fue del 88,3\%. En cuanto a las causas, el INEGi reportó que el $65,6 \%$ de las víctimas no denuncian por causas atribuibles a las autoridades como miedo a la extorsión, considerar el proceso una pérdida de tiempo, desconfianza en la autoridad, entre otras (INEGI 2014).

La Comisión Mexicana de Defensa y Promoción de los Derechos Humanos (смдрdн) y el Instituto Mexicano de Derechos Humanos (тмднр) en su informe del 2013, mencionaron que entre el $91 \%$ y el $98 \%$ de los crímenes permanecieron en la impunidad. Solo el 1,5\% del total de los delitos cometidos llegaron ante un juez. Esto viene ocurriendo desde hace varios años, por ejemplo, en un periodo de enero 2008-octubre 2012, del total de 8595 personas detenidas, tan solo el 3,2\% recibió sentencia condenatoria (Peace Brigades International-рві 2013).

Al mismo tiempo, frente al aumento de la criminalidad $y$ la desconfianza en las autoridades, se ha observado como muchos ciudadanos tienen una expectativa muy baja de los programas gubernamentales de seguridad pública y prevención criminal, por lo cual provoca el uso de sus propias medidas preventivas para no ser víctimas de un delito (Braakmann 2012). En algunos casos, la ciudadanía se ha organizado para diseñar mecanismos, tanto de prevención de la violencia como de castigo del delito; no obstante, en ocasiones son más violentos que los 
agresores mismos. Adicionalmente, se ha visto que estas medidas expresan rasgos socioeconómicos, socioculturales y psicosociales de las comunidades que los implementan (RomeroSalazar, Rujano y Romero 2009). En comunidades donde se ha logrado la autogestión de la seguridad, destacan dos grupos de estrategias que tienden a surgir de la preocupación y el interés vecinal: la "tercerización" y la "colectivización" de la seguridad. Mientras la tercerización encomienda la responsabilidad de la seguridad a entidades privadas, la colectivización supone una organización local para patrullaje y monitoreo de la seguridad comunitaria (Mollericona, Tinini y Paredes 2007).

Dado que actualmente la inseguridad en México y en particular, en el Estado de Morelos, es una de las problemáticas más graves a enfrentar y es la principal preocupación de la ciudadanía, tanto a nivel nacional $(64,5 \%)$, como en el propio Estado (58,1\%) (INEGI 2014), el objetivo principal del presente estudio es entender cómo se interrelaciona el incremento de la delincuencia con el actuar del gobierno y la experiencia ciudadana - víctimas directas e indirectas - y cómo se modula la percepción que se tiene de los niveles de inseguridad; observando además, que la mayoría de los trabajos previos se enfocan en relaciones de asociación bivariada entre variables $y$ que hasta ahora no se ha planteado un modelo explicativo de ecuaciones estructurales.

Los modelos de ecuaciones estructurales aportan una visión global de aspectos relevantes del fenómeno estudiado, en contraposición a otras herramientas estadísticas utilizadas en las ciencias sociales, que se centran en el análi- sis individual de cada factor. El uso de modelos es pertinente para este estudio, puesto que reduce la cantidad de información analizada, ya que su esencia es agrupar las relaciones entre un gran número de variables en unos pocos factores, permitiendo enfocarse en los aspectos fundamentales de la situación a explicar. Además, algunas de las variables no son medibles directamente y los modelos tienen la ventaja de carecer de error de medición (Batista y Coenders 2000).

Bajo los argumentos anteriores se ha formulado un modelo teórico hipotético para entender la realidad en el Estado de Morelos, asumiendo como guía el modelo ecológico de Bronfenbrenner (1979). El modelo planteado supone efectos indirectos o relaciones causales indirectas entre la victimización y la inseguridad percibida, a través de cuatro distintos factores moderadores: respecto a la perspectiva ciudadana sobre el actuar del gobierno: 1) el nivel de confianza en el desempeño o actuación de las autoridades y 2) la percepción de impunidad. Respecto a la experiencia ciudadana: 3) la expectativa sobre los programas gubernamentales de seguridad pública y prevención del delito y 4) las medidas preventivas asumidas por la ciudadanía victimizada o no. Al mismo tiempo, se plantea como hipótesis que la victimización ejerce un efecto directo sobre los factores moderadores; estos a su vez, ejercen efectos directos sobre la inseguridad percibida. El estudio de dichos factores $y$ sus relaciones, se asume bajo el supuesto de interacción multivariada causal directa e indirecta entre las variables del modelo estructural (figura 1). 


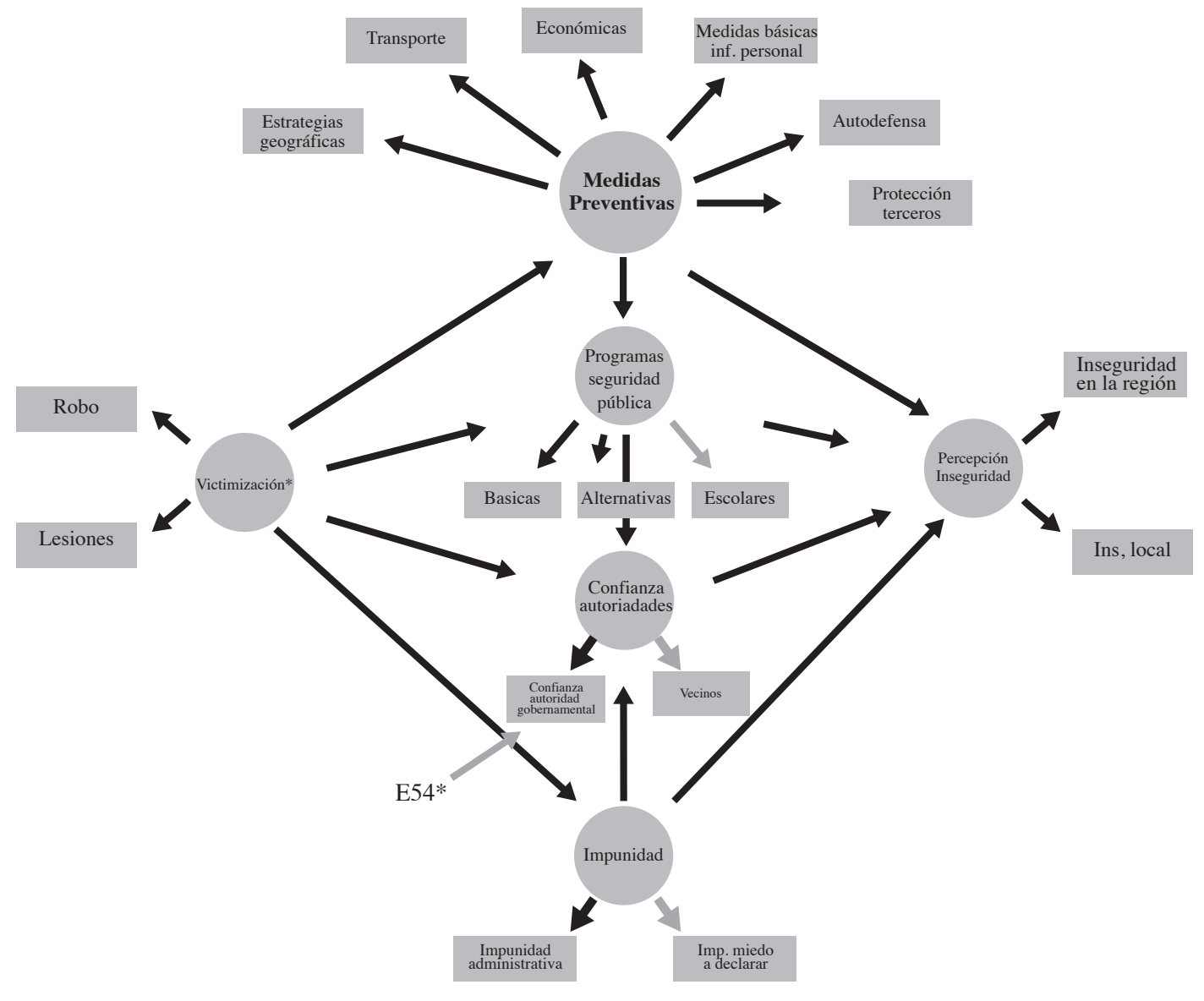

FIGURA 1

MODELO HIPOTÉTICO DE LA PERCEPCIÓN DE INSEGURIDAD EN LOS CIUDADANOS MORELENSES

Fuente: El modelo estructural hipotético fue desarrollado con EQS 6, a partir de la literatura científica y para ser corroborado con los datos de la encuesta Estatal de Percepción Ciudadana sobre la Violencia, la Delincuencia, la Inseguridad y la Dinámica Social e Institucional en el Estado de Morelos (2014).

METODOLOGÍA

DISEÑO

El estudio se realizó mediante un diseño de investigación transversal ex post facto, de tipo explicativo, a través del uso de modelos de ecuaciones estructurales.

\section{PARTICIPANTES}

La muestra fue de 8170 personas (4062 mujeres y 4082 hombres), que se seleccionaron a través de un muestreo "probabilístico multietápico", en función de grupos de edad, sexo y bajo el criterio de muestras representativas de cada uno de los 33 municipios del Estado de Morelos. Las edades oscilaron entre 
17 y 80 años, con una media de 46,06 y una desviación estándar de 9,5. El tamaño de la muestra permite evaluar el modelo con un coeficiente de determinación de .05 y un poder de .90 (Elashoff 2005).

\section{VARIABLES LATENTES, VARIABLES MANIFIESTAS Y SUS RELACIONES}

El modelo propuesto se basó en seis variables latentes o constructos teóricos medidos cada uno a través de dos o más indicadores manifiestos, que se refieren a las variables reportadas directamente por los participantes. A continuación se especifican las variables latentes, sus indicadores manifiestos y se describen sus relaciones teóricas hipotéticas de causalidad, planteadas en el diseño del modelo de ecuaciones estructurales (figura 1).

a) Victimización. Se refiere a si la persona fue víctima directa (que sufrió un ataque $o$ acto criminal) o víctima indirecta (son familiares o amigos cercanos a la víctima $y / 0$ viven las condiciones de violencia $y$ delincuencia del contexto social). Las víctimas directas se agruparon en dos tipos, en función de la manera como se perpetró el delito: sin agresión o con agresión. Se hipotetiza que la victimización produce un efecto directo en las Medidas Preventivas, en la Expectativa sobre Programas de Seguridad Pública, la Confianza en las Autoridades y la Impunidad Percibida. Produce también un efecto indirecto en la Percepción de Inseguridad.

b) Medidas Preventivas. La variable se compone de las medidas preventivas y las estrategias vs. la victimización. Se refiere a las diversas acciones que la ciudadanía ha tomado para protegerse $y$ evitar ser víctima directa de un delito o encontrarse en situaciones donde aumente el potencial de victimización. Se midió a través de tres tipos de medidas y tres tipos de estrategias:

$\diamond \quad$ Medidas preventivas: Básicas (dejar de dar información por teléfono, procurar andar acompañado, dejar de dar claves o datos por internet, dejar de dar información a desconocidos y utilizar identificador de llamadas), de Autodefensa (colocar bardas, rejas, cercas, conseguir un perro, dejar de dar claves o datos por internet, instalar alarmas en casa o trabajo, comprar o portar un arma, poner seguros adicionales en puertas/ventanas) y por terceros o Tercerización (comprar un seguro para casa, auto y/o vida, contratar seguridad personal y contratar seguridad privada en calle o colonia).

$\diamond \quad$ Estrategias vs. la victimización: Geográficas (ejemplos: no estacionar su vehículo en la calle, no salir muy temprano o de noche, no usar joyas o artículos caros, no caminar por calles oscuras y solitarias, no llevar celular a la vista, no traer más dinero del necesario y evitar zonas peligrosas de la localidad); Económicas (no llevar tarjetas de crédito o débito, no visitar parientes o amigos que viven lejos, no llevar dinero en efectivo y usar cajeros automáticos) y en Transporte (no tomar taxi ni usar transporte público).

La variable de Estrategias actúa como variabla interviniente entre la victimización y la inseguridad percibida $y$ tiene un efecto directo sobre la expectativa en los programas de seguridad pública.

c) Expectativa sobre los Programas de Seguridad Pública. Se trata de lo que la ciudadanía espera respecto a la capacidad de los proyectos gubernamentales vigentes sobre prevención y resguardo de la paz y el orden público, en el Estado de Morelos. Los programas fueron agrupados en tres categorías: Básicos (Prevención Social del Delito, Comunidad Segura, Seguridad Infantil, Prevención de la Mujer y Morelos Seguro), Alternativos (Justicia Alternativa, Sensores Ciudadanos y Escudo Centro) y Escolares (Escuela Segura y Mochila Segura). 
La expectativa actúa como variable interviniente entre la Victimización y la Inseguridad Percibida e influye directamente sobre la Confianza en las Autoridades y sobre la Inseguridad Percibida.

d) Confianza en Autoridades. Es la valoración de la ciudadanía de que los servidores públicos de las instituciones de Seguridad Pública están cumpliendo con sus obligaciones desde el nivel local hasta el nivel federal: Ronda Local y Vecinal, Policía Municipal, Policía Estatal, Policía Federal Preventiva, Ministerio Público, Mando Único, Procuraduría General de la República y Ejército.

Actúa como variable interviniente entre la Victimización y la Inseguridad Percibida y ejerce un efecto directo en la Inseguridad Percibida.

e) Impunidad Percibida. Se refiere a la creencia real o supuesta de que los criminales no están siendo procesados. Se plantean dos tipos de razones que la gente adjudica como causantes de la impunidad percibida: Administrativa (por la burocracia del sistema judicial) y por Miedo a las represalias (por parte de los criminales).

Actúa como variable interviniente entre la Victimización y la Inseguridad Percibida e influye directamente sobre la Confianza en las Autoridades y en la Percepción de Inseguridad.

f) Percepción de Inseguridad. Es la variable resultante y se refiere a la apreciación global de la ciudadanía sobre la condición de riesgo a ser víctima de la violencia y la delincuencia, acompañada de miedo o temor. Se consideró su percepción de seguridad en distintas demarcaciones geopolíticas, desde su calle, colonia, Municipio hasta el Estado de Morelos. Medida a través de la percepción de Inseguridad Regional e Inseguridad Local.

\section{INSTRUMENTO}

Se utilizó la Encuesta Estatal de Percepción Ciudadana sobre la Violencia, la Delincuencia, la Inseguridad y la Dinámica Social e Institucional en el Estado de Morelos (2014), que consta de 427 ítems agrupados en varias escalas, de las cuales se utilizaron solamente las siguientes:

1) Escala de Percepción de Seguridad: con 4 ítems tipo Likert, analiza la seguridad percibida desde la calle hasta el Estado. Con un coeficiente de fiabilidad ( $\alpha$ de Cronbach) obtenido de .76.

2) ¿Fue víctima de algún delito en el último año? Si la respuesta es positiva, se pregunta el Tipo de Victimización. Esta consta de una lista de 14 delitos principales. Con un coeficiente de fiabilidad obtenido de .99 .

3) Escala de Medidas de Prevención. Evalúa 14 distintas acciones que se agrupan en 3 categorías: Básicas, de Autodefensa y por terceros o Tercerización. Con un coeficiente de fiabilidad de .84 .

4) Escala de Estrategias vs. Victimización. Evalúa 14 distintas maniobras, que se agrupan en 3 categorías: Geográficas, Económicas y de Transporte. Con un coeficiente de fiabilidad de .85 .

5) Escala de Expectativas de Programas de Prevención. Evalúa 3 tipos de programas de reducción de la inseguridad: Básicos, Alternativos y Escolares. Cuenta con 10 ítems dicotómicos. Con un coeficiente de fiabilidad de .80 .

6) Escala de Confianza en Autoridades. Cuenta con 9 ítems tipo Likert. Con un coeficiente de fiabilidad de .90 .

7) Escala de Nivel de Impunidad Percibida. La escala cuenta con 12 ítems dicotómicos. Con un coeficiente de fiabilidad de .74.

\section{PROCEDIMIENTO}

Una vez definida la muestra representativa para cada entidad municipal del Estado de Morelos, se capacitó a un equipo de encuestadores que trabajaron en las distintas regiones del 
Estado. Se solicitó la colaboración voluntaria de los ciudadanos para contestar la encuesta $y$ se les garantizó confidencialidad y anonimato en sus respuestas. Las encuestas se realizaron a domicilio, con una duración aproximada de 45 minutos. Los encuestadores recibieron una compensación por sus servicios.

\section{MÉTODO ESTADÍSTICO}

Para el análisis estadístico de los datos obtenidos se utilizaron dos paquetes estadísticos, el spss 19.0 y el EQs 6.2, fijándose el nivel de significación estadística en .05. La consistencia interna de los instrumentos se estimó con el Alpha de Cronbach. Para determinar el grado de relación causal existente entre las variables de interés se diseñó un modelo con el método de ecuaciones estructurales. El modelo fue precedido por el análisis correlacional productomomento de Pearson. El análisis estructural se llevó a cabo a través de la contrastación del modelo explicativo hipotético de inseguridad percibida con el modelo resultante.

Para el contraste del modelo estructural se contemplaron tres Índices de Bondad de Ajuste Absoluto: a) Error Medio Cuadrático de Aproximación a Variables de la población (RAMSEA por sus siglas en inglés), cuyo valor ideal es $\leq .05$; b) el Índice de Bondad de Ajuste de Joreskog (GFI), el cual se interpreta como una proporción de varianza explicada a $R^{2}$ en regresión múltiple, en este sentido un Gri de 1,0 indicaría ajuste perfecto y el modelo explicaría el $100 \%$ de la varianza en los datos observados (el criterio aceptado es de $\geq .90$ ); c) el Índice de Bondad Ajustado de Joreskog (AGFI) que ajusta el GFI, asumiendo los grados de libertad en el modelo a prueba. Además, se consideraron cuatro índices de ajuste comparativo, que contrastan un modelo hipotético con el modelo de independencia. Este último se refiere al modelo nulo, que es aquel en el que todas las correlaciones son próximas a cero, siendo el modelo saturado el contrario con correlaciones perfectas. Los Índices de Ajuste Comparativo que se analizaron para este estudio fueron: 1) Índice de Ajuste Normado de Bentler-Bonnet (NFI), que se interpreta como un porcentaje de incremento en la bondad de ajuste sobre el modelo nulo, esto supondría que un modelo hipotetizado ajusta
90\% mejor que el nulo; 2) Índice No Normado de Bentler-Bonnet (NNFI), que toma valores inferiores a cero y si las puntuaciones son superiores a .90, el ajuste es adecuado; 3) Índice Comparativo de Ajuste de Bentler ( $\mathrm{CFI}$ ), con valores entre 0 y 1 (valores de $\geq .90$ son ajuste razonable); 4) Índice de Ajuste de Incremento de Bollen (IFI) que reintroduce un factor de escala en el rango de 0 a 1 (entre más alto indica mejor ajuste).

\section{LIMITACIONES}

La presente investigación empleó un procedimiento metodológico riguroso, sin embargo, existen ciertas limitaciones que resulta necesario mencionar: se trata de un estudio transversal y por lo tanto, la atribución de asociación causal entre las variables dependientes e independientes requiere corroborarse longitudinalmente. Por otro lado, existe una gran cantidad de factores que probablemente también estén implicados en la relación entre Victimización e Inseguridad percibida, que no fueron explorados en este estudio. En este sentido, cuantos más factores negativos influyan en la percepción de la ciudadanía, más difícil es recobrar la confianza y cambiar la percepción de inseguridad a seguridad.

\section{RESULTADOS}

Se presentan primero los resultados del análisis descriptivo (tabla 1), posteriormente se presenta la matriz de correlaciones (tabla 2) y finalmente, el modelo resultante de ecuaciones estructurales (figura 2). Los datos socio-demográficos de los participantes en función de su calidad de víctimas directas o indirectas mostraron que el 12,2\% reportó haber sido víctima directa de uno o más delitos, mientras que el 87,8\% fue víctima indirecta (vive condiciones de violencia y criminalidad del contexto social). De las víctimas directas, el 50,4\% fueron mujeres; en cuanto a su edad, el grupo etario con más víctimas fue de jóvenes de entre 20 y 40 años (37,2\%). El $54 \%$ reportó tener un empleo remunerado y el 40,1\% vive en Cuernavaca o Cuautla. El 83,9\% de las víctimas directas y el 75,3\% de las víctimas indirectas percibieron un nivel de seguridad global bajo. Respecto a las expectativas de los 
programas gubernamentales de seguridad, el $33,9 \%$ de las víctimas directas junto con $21,5 \%$ de las víctimas indirectas, tuvieron muy bajas expectativas. Sin embargo, el 45,3\% de las víctimas indirectas y el $28 \%$ de las víctimas directas revelaron no conocer los programas.

En cuanto a la confianza en las autoridades, el $69,6 \%$ de las víctimas directas y el $64,6 \%$ de las víctimas indirectas, reportaron poca o baja confianza. El nivel de impunidad percibido fue bastante alto: el 57,9\% de las víctimas directas y el $49,9 \%$ de las víctimas indirectas, consideraron que el nivel de impunidad en Morelos es muy alto. En cuanto a las razones por las cuales las víctimas directas no denunciaron y de esta manera fomentaron la impunidad, la más común fue pensar que era una "pérdida de tiempo" $(42,5 \%)$, en segundo lugar, consideraron que "los trámites son largos y difíciles" $(34,2 \%)$ y en tercer lugar, no denunciaron por seguridad familiar o miedo al agresor $(19,2 \%)$ de las víctimas directas. Finalmente, en cuanto a las medidas preventivas, el $58,4 \%$ de las víctimas directas $y$ el $45,3 \%$ de las víctimas indirectas, han implementado de 7 a 14 medidas distintas.

TABLA 1

DATOS SOCIODEMOGRÁFICOS Y DESCRIPTIVOS DE LA MUESTRA ESTUDIADA

\begin{tabular}{|c|c|c|c|c|c|c|}
\hline & \multicolumn{2}{|c|}{$\begin{array}{c}\text { VÍCTIMAS } \\
\text { INDIRECTAS }\end{array}$} & \multicolumn{2}{|c|}{$\begin{array}{l}\text { VÍCTIMAS } \\
\text { DIRECTAS }\end{array}$} & \multicolumn{2}{|c|}{ TOTAL } \\
\hline & $\mathrm{N}$ & $\%$ & $\mathrm{~N}$ & $\%$ & $\mathrm{~N}$ & $\%$ \\
\hline \multicolumn{7}{|l|}{ SEXO } \\
\hline Femenino & 3582 & 49,9 & 504 & 50,4 & 4086 & 50 \\
\hline Masculino & 3588 & 50,1 & 496 & 49,6 & 4084 & 50 \\
\hline \multicolumn{7}{|l|}{ EDAD } \\
\hline 16 a 19 años & 2200 & 30,7 & 226 & 22,6 & 2426 & 29,7 \\
\hline 20 a 40 años & 2061 & 28,7 & 372 & 37,2 & 2433 & 29,7 \\
\hline 41 a 60 años & 1467 & 20,5 & 237 & 23,7 & 1704 & 20,8 \\
\hline 61 o más & 1441 & 20,1 & 165 & 16,5 & 1607 & 19,7 \\
\hline \multicolumn{7}{|l|}{ REGIÓN } \\
\hline Cuerna. y Cuautla & 2756 & 38,5 & 401 & 40,1 & 3157 & 38,6 \\
\hline Resto del Estado & 4414 & 58,6 & 599 & 59,9 & 5013 & 61,3 \\
\hline \multicolumn{7}{|l|}{ OCUPACIÓN } \\
\hline Remunerada & 2815 & 39,3 & 544 & 54,4 & 3359 & 41,1 \\
\hline No remunerada & 2078 & 28,9 & 219 & 21,9 & 2297 & 28,1 \\
\hline Estudiante & 2277 & 31,8 & 237 & 23,7 & 2514 & 30,8 \\
\hline \multicolumn{7}{|c|}{ NIVEL DE SEGURIDAD } \\
\hline Baja & 5400 & 75,3 & 839 & 83,9 & 6239 & 76,4 \\
\hline Media & 1641 & 22,9 & 156 & 15,6 & 1797 & 22 \\
\hline Alta & 129 & 1,8 & 5 & 0,5 & 134 & 1,6 \\
\hline \multicolumn{7}{|c|}{ EXPECTATIVAS DE PROGRAMAS DE SEGURIDAD } \\
\hline Baja & 159 & 21,5 & 339 & 33,9 & 1878 & 23 \\
\hline Regular & 297 & 4,1 & 72 & 7,2 & 369 & 4,5 \\
\hline Alta & 2083 & 29,1 & 309 & 30,9 & 2392 & 29,3 \\
\hline sin dato* & 3251 & 45,3 & 280 & 28 & 3531 & 43,2 \\
\hline \multicolumn{7}{|c|}{ CONFIANZA EN AUTORIDADES } \\
\hline Baja & 4629 & 64,6 & 696 & 69,6 & 5325 & 65,2 \\
\hline Regular & 2352 & 32,8 & 281 & 28,1 & 2633 & 32,2 \\
\hline Alta & 189 & 2,6 & 23 & 2,3 & 212 & 2,6 \\
\hline \multicolumn{7}{|c|}{ IMPUNIDAD PERCIBIDA } \\
\hline Baja & 833 & 11,6 & 91 & 9,1 & 924 & 11,3 \\
\hline Media & 2069 & 28,9 & 279 & 27,9 & 2348 & 28,7 \\
\hline Alta & 3574 & 49,9 & 579 & 57,9 & 4153 & 50,8 \\
\hline \multicolumn{7}{|c|}{ MEDIDAS PREVENTIVAS } \\
\hline 0 a 2 & 1337 & 18,6 & 98 & 9,8 & 1437 & 17,6 \\
\hline 3 a 6 & 2584 & 36 & 318 & 31,8 & 2902 & 25,5 \\
\hline 7 a 14 & 3249 & 45,3 & 585 & 58,4 & 3833 & 46,9 \\
\hline
\end{tabular}

Fuente: Los datos fueron obtenidos a partir de los resultados de la Encuesta Estatal de Percepción Ciudadana sobre la Violencia, la Delincuencia, la Inseguridad y la Dinámica Social e Institucional en el Estado de Morelos (2014).

Nota*: Las personas que desconocen de la existencia de los programas no contestaron a la pregunta de nivel de expectativa de los mismos. 
En la tabla 2 se presenta la matriz de correlaciones de Pearson con las variables estudiadas. Se observa que la percepción de inseguridad, tanto a nivel local como regional, se asoció de manera muy débil con la mayoría de las variables. La inseguridad regional no correlacionó de manera significativa con los programas gubernamentales de prevención. Las correlaciones significativas de Inseguridad Regional fueron con Estrategias Geográficas $(r=.172, p<.01)$, Estrategias Económicas $(r=.152, p<.01)$, Confianza en Autoridades $(r=-.072, p<.01)$, Impunidad Administrativa $(r=.081, p<.01)$. Con la Inseguridad Local la mayoría de las variables correlacionan significativamente, excepto los Programas Preventivos. Algunas de las correlaciones relevantes de la Inseguridad Local son: Estrategias de Transporte $(r=.139, p<.01)$; Estrategias Económicas $(r=.157, p<.01)$; Impunidad Administrativa $(r=.084, p<.01)$ entre otras. En el caso de la asociación entre las variables de los mismos grupos mostraron en general, niveles altos de correlación. 


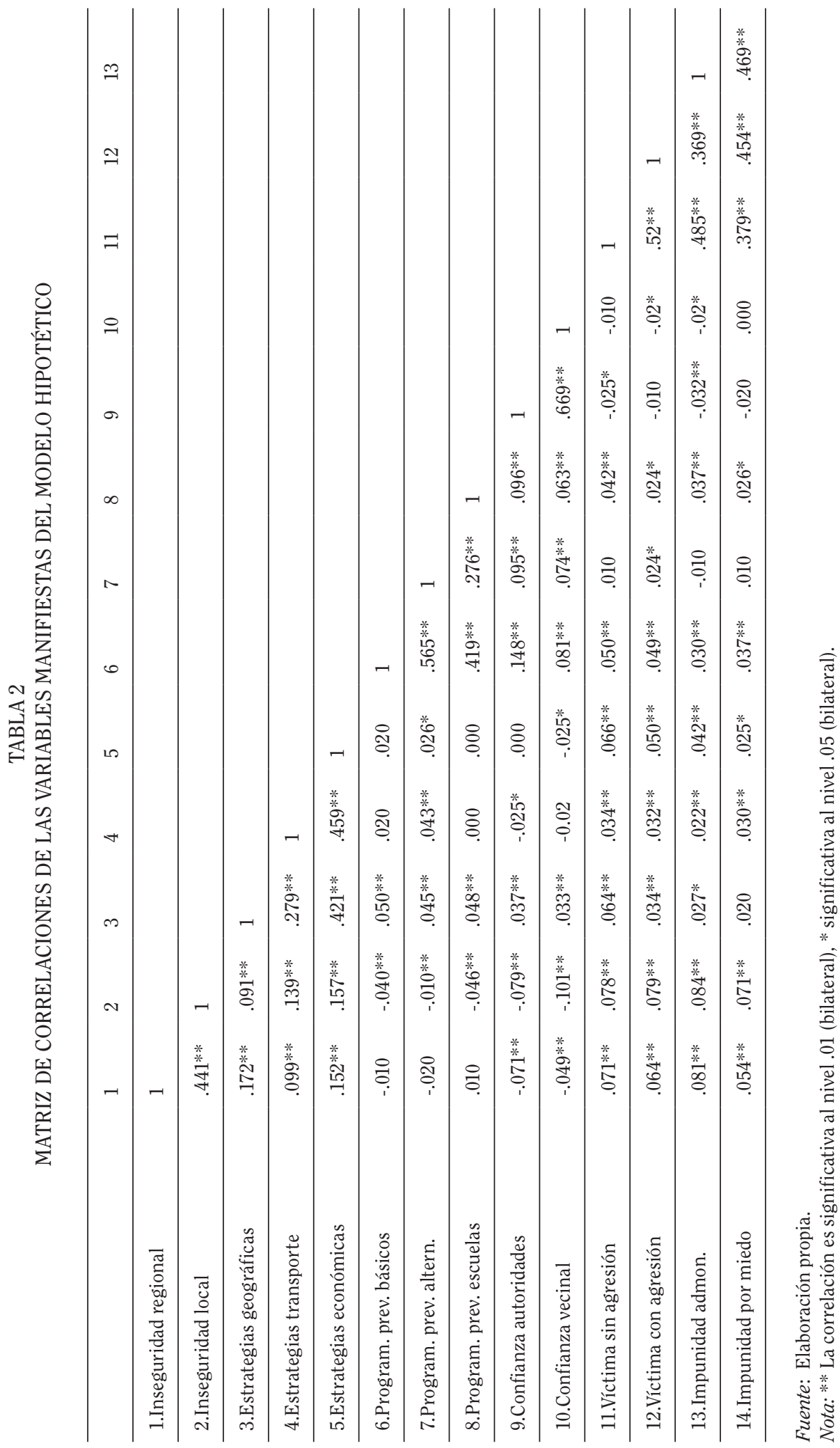


A partir de los resultados del análisis correlacional, se procedió a calcular el modelo resultante de ecuaciones estructurales para analizar la existencia de relaciones causales, a través de los efectos directos e indirectos de las variables de interés. Para cada variable observable se calculó la saturación en el factor correspondiente, así como, su error de medición y se determinó su relación dentro del modelo (figura 2).

El modelo calculado ajustó bien al modelo hipotético, como indican los siguientes índices: $\mathrm{cFI}=.91$, NNFI $=.89$, GFI $=.96$, AGFI $=.94$, IFI $=.91$ y RMSEA $=.05$ (figura 2). El modelo eXplicó el $22,6 \%$ de la varianza de Percepción de Inseguridad y confirmó la existencia de efectos indirectos de la Victimización en la Percepción de Inseguridad, a través de la im- punidad $(\beta=.78, \mathrm{p}<.001)$, de los Programas de Seguridad Pública $(\beta=.09, \mathrm{p}<.001)$, de las Medidas de Prevención $(\beta=.20, \mathrm{p}<.001)$, así como de la confianza en las autoridades $(\beta=-.16, p<$ $.01)$. Al mismo tiempo, los resultados mostraron un efecto positivo directo de las Medidas de Prevención $(\beta=.33, p<.001)$ y la Impunidad $(\beta=.17, \mathrm{p}<.001)$ en la Percepción de Inseguridad. Mientras que el efecto directo de la confianza en las autoridades $(\beta=-.20, \mathrm{p}<.001)$ $y$ los programas de seguridad pública $(\beta=-.11$, $\mathrm{p}<.001)$ en la percepción de inseguridad fue negativo. También se observaron efectos positivos directos de los programas de seguridad pública en la confianza en las autoridades $(\beta=.15, \mathrm{p}<.01)$ y efectos negativos directos de la impunidad en la confianza en las autorida$\operatorname{des}(\beta=-.10, \mathrm{p}<.01)$. 


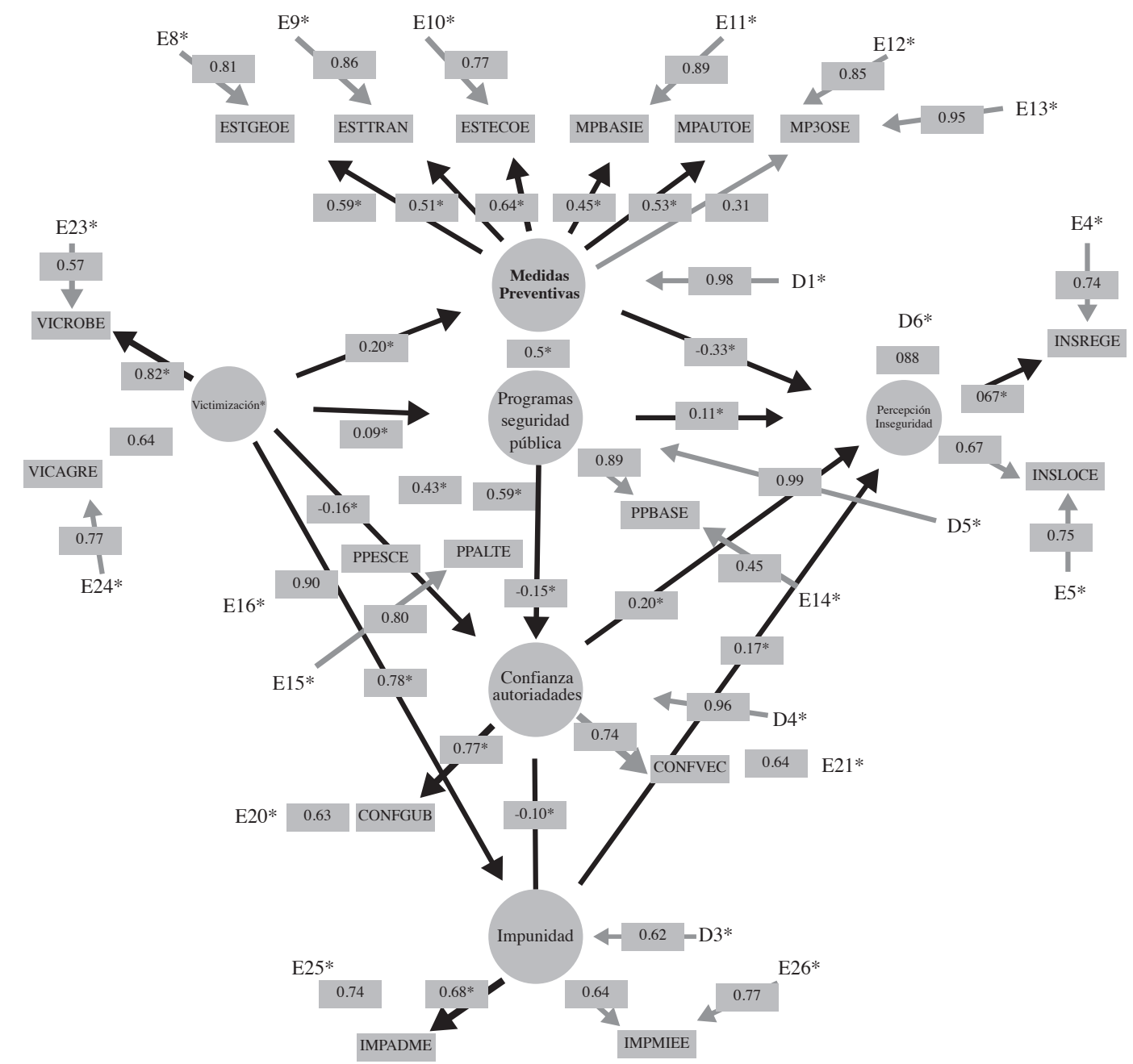

Figure X: EQS 6 modelo medidas preventivas 26 Chi Sq. $=667.70 \mathrm{P}=0.00 \mathrm{CFI}=0.91 \mathrm{RMSEA}=0.05$

FIGURA 2

MODELO EXPLICATIVO DE LA PERCEPCIÓN

DE INSEGURIDAD EN LA POBLACIÓN DE MORELOS

Fuente: Elaboración propia. 
Es importante destacar los coeficientes que determinaron la intensidad de la relación entre variables de gran interés para este estudio, como fue el caso de la relación positiva directa entre Victimización e Impunidad $(\beta=.78)$, que confirmó que la Victimización influye directamente sobre la percepción de Impunidad. Esto sugiere que las consecuencias que la experiencia de Victimización tuvo sobre el individuo, no fueron solo relativas el acto delictivo, sino también al proceso posterior en el que probablemente, la denuncia de la víctima no procedió correctamente y el culpable quedó impune. A su vez, la impunidad se relacionó positivamente con la Percepción de Inseguridad $(\beta=.17)$, corroborando que el incremento de la impunidad percibida, no hizo más que aumentar inevitablemente la sensación de inseguridad. También se destaca la relación positiva directa entre Victimización y Medidas Preventivas $(\beta=.20)$, es decir, que las víctimas directas tendieron a llevar a cabo más medidas preventivas que las víctimas indirectas, probablemente como consecuencia de su experiencia traumática. Mientras que la relación entre Victimización y Confianza en las Autoridades fue negativa $(\beta=-.16)$, esto supone que la Victimización redujo la Confianza en las Autoridades. La confianza a su vez tuvo una relación negativa directa con la Percepción de Inseguridad $(\beta=-.20)$, que refiere que a menor confianza mayor inseguridad percibida, especialmente cuando se ha sido víctima directa de la delincuencia.

\section{DISCUSIÓN}

El objetivo principal de este estudio fue diseñar $y$ contrastar un modelo de ecuaciones estructurales, en el que, bajo un modelo teórico, se integraron variables relacionadas con la situación actual de violencia y delincuencia en el Estado de Morelos, las instituciones gubernamentales de seguridad y los ciudadanos; para explicar posibles factores que junto con la victimización, han dado pie al incremento de la inseguridad percibida en los últimos años. Para sustentar el planteamiento del modelo, primero se observa que los resultados descriptivos revelaron que la mayoría de los morelenses percibieron un nivel muy alto de inseguridad.
Sin embargo, solo el $12 \%$ de los morelenses encuestados fueron víctimas directas de actos delictivos en el último año. Esto corroboró uno de los principales argumentos, de que no se tiene que ser víctima directa de actos delictivos para percibir la pérdida de la seguridad (Chadee y NgYing 2013, Hipp 2010, Zimring y Hawkins 1997, INEGI 2014). La diferencia entre cantidad de victimización e inseguridad percibida, como se había predicho, confirmó la necesidad de profundizar su estudio a través de un modelo estructural con factores moderadores entre dichos fenómenos psicosociales.

Los factores moderadores planteados en el modelo, se basaron en los supuestos teóricos surgidos de las interacciones ampliamente estudiadas entre victimización e inseguridad percibida (Bailey y Flores-Macías 2007, Chadee y NgYing 2013, Hipp 2010, Zimring y Hawkins 1997); entre inseguridad y confianza en las autoridades (Acero 2005, Dammert 2012, Ruíz 2007); entre victimización e impunidad (Hernández 2008, Matus 1998); entre victimización, inseguridad y medidas preventivas (Mollericona et ál. 2007, Braakmann 2012) y entre desconfianza e impunidad (Mier Garza 2010).

Todo lo anterior dio sustento al modelo estructural diseñado para este estudio, al confirmar la existencia de factores que intervienen incrementando la percepción de inseguridad, como la desconfianza en las autoridades, la percepción de un alto nivel de impunidad, junto con el desconocimiento o la baja expectativa de los programas de seguridad pública y la necesidad de protegerse por el temor a ser victimizado. Es decir que, un contexto social con altos índices de violencia $y$ delincuencia, se traduce en un contexto psicológico de temor generalizado, por el riesgo subjetivo de convertirse en víctima, a través de factores que moderan dicha relación.

El modelo resultante concordó con las relaciones bidireccionales expuestas en estudios anteriores $y$ adicionalmente permitió establecer relaciones de causalidad entre las variables de interés al confirmar las hipótesis de que la impunidad percibida, la confianza en las autoridades, las medidas preventivas y los programas de seguridad pública fungen como moderadores 
entre el aumento de la victimización y la inseguridad percibida en el Estado de Morelos.

En paralelo, el modelo ratificó que la victimización ejerce una fuerte influencia directa sobre la impunidad percibida. A su vez, el modelo mostró que la impunidad percibida lleva a la ciudadanía a sentir más inseguridad, tanto regional como local. La impunidad también tuvo un efecto directo en la confianza en las autoridades; esto significa que el aumento en la percepción de impunidad, disminuyó su confianza en estas. Como se ha señalado en otros estudios, la impunidad se asocia en gran parte a la baja eficacia institucional de los cuerpos policiacos (Carbonell 2006); asimismo, se asocia al incremento de la delincuencia, dado que los criminales aprenden que pueden ejecutar sus acciones pues difícilmente serán castigados (Romero-Salazar, Rujano y Romero 2009). Además, sugiere que la experiencia de las víctimas - tanto directas como indirectas-, con el sistema de justicia no ha sido satisfactoria y por el contrario, ha promovido su apreciación negativa del sistema, manifestando abusos, arbitrariedades, ilegalidad y corrupción en las acciones de las autoridades. Esto va en línea con lo reportado por la Comisión Mexicana de Defensa y Promoción de los Derechos Humanos (смдpd) y el Instituto Mexicano de Derechos Humanos y Democracia (тмднd) (2013), Jusidman (2010), Mier Garza (2010), Falconi (2007), entre otros.

En la evaluación de la ciudadanía morelense, resaltó el efecto directo de la baja confianza en las autoridades y en el aumento de la inseguridad percibida. El hecho de poner en duda el que las instituciones encargadas de la prevención del delito y de brindar la seguridad necesaria para una vida digna, cumplan con sus funciones, genera incertidumbre; a pesar de las estrategias implementadas por el gobierno, como involucrar a la policía federal y al Ejército, en la seguridad de Morelos. Se observa que los ciudadanos se mostraron profundamente preocupados, no solo por la enorme vulnerabilidad ante las altas tasas de criminalidad (en particular por los delitos violentos), sino también por la insatisfactoria respuesta gubernamental ante el incremento de actos delictivos.
Ambos factores -impunidad y desconfianza-, influyeron de forma determinante en el incremento de la percepción de inseguridad y generando un círculo vicioso, por ejemplo, la ausencia de denuncias, solo ha acrecentado el problema. Por el contrario, se sugiere que si las instituciones desempeñaran sus funciones adecuadamente y cuidaran de la aplicación del estado de derecho (Carbonell, Orozco y Vázquez 2002), aumentaría la confianza de la ciudadanía y como consecuencia, disminuiría la inseguridad percibida. La desconfianza también se materializa en debilidad institucional, lo que hace reprochable al Estado la falta de control (corrupción) y la impunidad (cultura del temor), generando que la sociedad perciba desprotección por parte del Estado, sienta que la ley es ineficaz y que las autoridades no actúan con la prontitud y eficacia necesarios (Navarro 2012). Por estas razones, es fundamental generar estrategias para reducir al máximo la impunidad, fomentar la denuncia ciudadana $y$ construir cuerpos policiacos fiables, que reconstruyan la confianza de la ciudadanía en ellos. Algunos recursos pueden ser, acercarse a la población a través de reintroducir a la policía comunitaria, fomentar la participación y empoderamiento de la ciudadanía, cambiar las acciones represivas y basadas en la "mano dura" por acciones preventivas, entre otros. Definitivamente, los resultados apuntan a que se debe trabajar intensamente en mejorar el sistema de justicia, pues la ciudadanía morelense no confía en este.

Además, la desconfianza en las instituciones de seguridad pública junto con la sensación generalizada de impunidad, no solo confirman una crítica por no atender las demandas sociales, que fomentan una imagen negativa de los servidores públicos y las instituciones gubernamentales de seguridad, sino como afirma la onu (1985), estas condiciones pueden llevar a creer que las autoridades están involucradas con los criminales o en alguna forma son responsables $y$ hasta culpables del nivel de delincuencia que se vive. Esto además coincide con otros estudios realizados en México (Bailey y Flores-Macías 2007; Carbonell 2006 y Hernández 2008) y esboza una combinación catastrófica que ha ido en aumento en 
los últimos años, como se ha observado con base en las estadísticas de la percepción ciudadana en México (Instituto Nacional de Estadística y Geografía 2011, 2012, 2013 y 2014).

A esto se une el hecho de que apenas la mitad de la población reportó conocer los programas oficiales de Seguridad Pública (Básicos: Prevención Social del Delito, Comunidad Segura, Seguridad Infantil, Prevención de la Mujer y Morelos Seguro; Alternativos: Justicia Alternativa, Sensores Ciudadanos y Escudo Centro; y Escolares: Escuela Segura y Mochila Segura). Lo que sugiere que los beneficios de dichos programas no están llegando a la población morelense, por lo tanto, no se están atendiendo sus necesidades de seguridad $y$ en consecuencia, no se puede esperar que la gente valore algo que no conoce.

De las personas que conocen los programas gubernamentales de prevención o han sido sus beneficiarios, las víctimas directas, tuvieron una expectativa más alta que el resto de la ciudadanía. Los resultados descriptivos revelaron que independientemente de la victimización, los participantes reportaron cierta ambivalencia ante dichos programas, puntuándolos muy alto o muy bajo. Al mismo tiempo, se confirmó que la satisfacción con los programas de seguridad redujo el sentimiento de inseguridad principalmente de quienes han sido victimizados. Por su parte, las víctimas indirectas reportaron una muy baja expectativa de los mismos. Esto se puede ver al analizar el modelo, el cual reveló un efecto indirecto de la victimización sobre la inseguridad, a través de las expectativas ciudadanas respecto a los programas de seguridad pública. Estos resultados van en línea con explicaciones anteriores, que señalan que no ha habido la respuesta esperada de las instituciones para reparar los abusos y sobre todo para evitar que estos ocurran (смDPDH y ІмDнD 2013; Hernández 2008). Conjuntamente, se confirmó un efecto positivo directo de la expectativa de los programas sobre la confianza en las autoridades. Es decir, que aunque no ha habido una respuesta esperada de las autoridades, si la ciudadanía tuviera una perspectiva positiva de los programas, esto contribuiría a incrementar la confianza en las autoridades y las instituciones de seguridad, como muestran los resultados.

Los hallazgos anteriores apuntan a trabajar en la continuación, fortalecimiento $y$ mejora de los programas gubernamentales de prevención y seguridad pública, para ayudar a disminuir la percepción de inseguridad $y$ aumentar la confianza en las autoridades. Además, el desconocimiento de la existencia de dichos programas, indica que se deberían generar más estrategias de difusión y acercamiento a la población, además de robustecer sus acciones preventivas, para que no sean solo devaluados o considerados elefantes blancos, sino entidades gubernamentales funcionales $y$ eficaces, que lleven a confiar en las autoridades $y$ haciendo que realmente la ciudadanía utilice $y$ se enorgullezca de contar con ellos.

En cuanto al despliegue de medidas preventivas y estrategias contra la victimización, tanto las víctimas directas como las indirectas, reportaron llevar a cabo un número muy alto de medidas; es decir, que no se tiene que ser víctima directa para verse en la necesidad de desplegar sus propias medidas de protección. Empero, las medidas o estrategias que la población en general ha asumido, parecieran ser solo paliativos que no disminuyen la percepción de inseguridad ni el miedo generalizado. Por el contrario, según el modelo, quienes más estrategias han implementado en su vida cotidiana, parecen ser quienes más inseguros y más temor reportan. Es decir, que aunque la ciudadanía asume cierta responsabilidad sobre su propia seguridad ante la falta de respuesta de las autoridades, estas medidas no logran modificar su percepción de inseguridad. Esto puede deberse al tipo de medidas implementadas, mayoritariamente de corte represivo, violento e individualista, que confirma que este tipo de medidas no incide en la disminución de la delincuencia ni la seguridad (RomeroSalazar et ál. 2009), mucho menos en el mejoramiento del bienestar y la calidad de vida de los ciudadanos.

Es fundamental que el combate a la delincuencia, vaya acompañado de importantes cambios en los factores que juegan un papel en el incremento del temor de la ciudadanía, 
a partir del fortalecimiento de la seguridad pública ciudadana y el estado de derecho, en todos los niveles de jurisprudencia del gobierno. Además, es más factible que este esfuerzo trascienda si es ampliamente apoyado por la ciudadanía, bajo el argumento de la coproducción de la seguridad (onu-habitat 2009). No obstante, de no mejorar la confianza en las autoridades, disminuir la impunidad, acercar los programas de seguridad a la gente $y$ fomentar la corresponsabilidad ciudadana, es muy probable que cada vez sea más evidente lo que se ha señalado antes, el deterioro de la democracia (Bailey y Flores-Macías 2007, Dammert 2010), la reducción del respeto por los derechos humanos (Arslanián 1998), junto con la devaluación y pérdida de la legalidad (Falconi 2007, Carbonell et ál. 2002) y por supuesto, la pérdida del bienestar, entre otras graves consecuencias.

Adicionalmente, los niveles tan altos de inseguridad percibida en Morelos, junto con el nivel de desconfianza en las autoridades, el gran desconocimiento de programas gubernamentales y la fuerte percepción de impunidad, puede llevar a la reducción o pérdida de la cohesión y la estabilidad social, como se ha advertido en otros contextos (Dammert 2010, Amerio y Roccato 2005, Mollericona et ál. 2007). Como plantean Guerrero y colaboradores (1994), esto pone en juego la convivencia pacífica y armónica, promoviendo comportamientos violentos entre la ciudadanía, especialmente si las autoridades respaldan el uso de la violencia con sus propias acciones; $y$ de continuar así, pueden llegar a generar una "cultura de la violencia" (Ugaz 1999).

El modelo desarrollado en este estudio, apunta a establecer algunas líneas esperanzadoras en donde podría mejorar la percepción de la inseguridad si realmente aumentara la confianza en las autoridades por su buen desempeño, eficacia en el cumplimiento de sus obligaciones y acercamiento a la ciudadanía. Ninguna campaña mediática que pretenda mejorar la imagen del Estado es efectiva si no llega a una ciudadanía, que comience a sentirse segura y libre para transitar en su país. En este sentido, se propone fortalecer dos líneas fundamentales:
1) Enfocada en la prevención del delito con programas de capacitación a los cuerpos policiacos locales. Fomentar su inserción y convivencia con las comunidades que protegen. Ampliar las vías de comunicación entre la ciudadanía y las autoridades, para disminuir la impunidad por falta de denuncias e incrementar la confianza en las autoridades; por ejemplo, a través de líneas telefónicas de emergencia con atención efectiva.

2) Enfocada en la atención a las víctimas, tanto directas como indirectas; contemplando desde la capacitación del personal que acude al llamado de auxilio, hasta la fiscalización en el funcionamiento transparente de los ministerios públicos, de tal manera que quien ha sido vulnerado en sus derechos no vuelva a serlo al acercarse a las autoridades gubernamentales, sino que encuentre realmente la atención $y$ respuesta ante sus demandas.

La seguridad otorgada por las autoridades se debe enfocar a una dimensión pro-activa de prevención, como se ha planteado antes (González, López y Yáñez 1994), y como teóricamente establecen la mayoría de los estatutos de los programas gubernamentales de Seguridad Pública. Esto permite actuar sobre las causas y no solo sobre los síntomas (Hurtado de Molina $2007 y$ onu 1968). Asimismo, debe buscar involucrar a la comunidad, a través de estrategias como la policía comunitaria y de proximidad (Rico y Chinchilla 2002). Sin embargo, nuestros resultados en línea con otros estudios (Reyes 2007, Bonastra et ál. 2006), muestran que en la perspectiva de la ciudadanía esto no se ha logrado. La prevención y el fortalecimiento de la seguridad ciudadana fomentan el bienestar social y la calidad de vida (ops 2002 y Acero 2005), ayudando a establecer las condiciones psicosociales básicas para el desenvolvimiento de las potencialidades humanas, las libertades, la solidaridad, la participación democrática y la creatividad (pNud 2013 y onu-HABITAT 2009), a través de una cultura de la paz y la convivencia (Guerrero et ál. 1994). 
En síntesis, el presente estudio resulta estratégico al brindar elementos para el diseño de políticas públicas y programas de seguridad pública ciudadana, que no solo incidan en la reducción de la percepción de inseguridad, sino que contribuyan a generar una mayor calidad de vida, asimismo, se busca una mayor participación ciudadana para enfrentar la situación que vive el país. Es importante resaltar que el resultado que debe generar un Estado eficaz y honesto, es seguridad para sus ciudadanos (Carbonell et ál. 2002).

\section{REFERENCIAS}

\section{LIBROS}

Acero, Hugo. 2005. "La seguridad ciudadana. Una responsabilidad de los gobiernos locales". Ciudad y seguridad en América Latina, editado por Lucía Dammert y Guatavo Paulsen, 133-150. Chile: FLacso.

Batista, Joan Manuel y Germà Coenders. 2000. "Modelos de Ecuaciones Estructurales". En Cuadernos de Estadística 6. Madrid: Editorial La Muralla sa.

Bonastra, Quim; Celeste Arella; Gabriela Rodríguez y Pedro Fraile. 2006. Paisaje ciudadano, delito y percepción de la inseguridad. Investigación interdisciplinaria del medio urbano. Madrid: Dykinson.

Carbonell, Miguel; Wistano Orozco y Rodolfo Vázquez. 2002. Estado de derecho. Concepto, fundamentos y democratización en América Latina. México, DF: Siglo xxi Editores.

Comisión Mexicana de Defensa y Promoción de los Derechos Humanos (смдрdн) e Instituto Mexicano de Derechos Humanos y Democracia (тмднр). 2013. Acceso a la justicia en México: La constante impunidad en casos de violaciones a derechos humanos. México: смDPDH-IMDHD. Acceso el 12 de junio de 2014. https:// www.iccnow.org/documents/Access_to_ Justice_in_Mexico_-_Spanish.pdf

Consejo Ciudadano para la Seguridad Pública y Justicia Penal A. C. 2013. La violencia en los municipios y las entidades federativas de México 2013. México: Editor pbsiar. Acceso el 4 de marzo de 2014: http://editor.pbsiar.com/upload/ PDF/2014_02_05_seguridad_justicia_y_ paz_municipios_violentos_2013.pdf

Dammert, Lucía. 2012. Fear and crime in Latin America: redefining state-society relations. New York: Rutledge.

Elashoff, Janet. 2005. Query Advisor Version 6.0 User's guide. Los Angeles: Statistical Solutions Ltd.

Falconi, Juan. 2007. Seguridad jurídica. Ecuador: Corporación de Estudios y Publicaciones.

González, Samuel; Ernesto López y José Arturo Yáñez. 1994. Seguridad pública en México. Problemas, perspectivas y propuestas. México: Publicaciones Universidad Nacional Autónoma de México.

Hurtado de Molina, Julián. 2007. La prevención del delito y del riesgo. 1era. Edición. Madrid: Dykinson.

Mollericona, Juan Yhonny; Ninoska Tinini y Adriana Paredes. 2007. La seguridad ciudadana en la ciudad de El Alto: fronteras entre el miedo y la acción vecinal. La Paz: Programa de Investigación Estratégica en Bolivia.

Polit, Denise y Beck Hungler. 2010. Nursing research: principles and methods. Philadelphia: Lippincott.

Rico, José María y Laura Chinchilla. 2002. Seguridad ciudadana en América Latina. México DF: Siglo xxI.

Roché, Sebastian. 1998. Sociologie politique de insecurité, violences urbaines, inégalités et globalisation. París: Presses Universitaires de France 1998.

Ugaz, José. 2002. Grandes urbes e inseguridad ciudadana. Seguridad ciudadana y derechos humanos. Lima: 327-343.

Urie Bronfenbrenner. 1979. The ecology of human development. Cambridge, Massachusetts: Harvard University Press.

Zimring, Franklin E. y Gordon Hawkins. 1997. Crime is not the problem. Oxford: Oxford Press. 
PUBLICACIONES PERIÓDICAS

Amerio, Piero y Michele Roccato. 2005. "A predictive model for psychosocial reactions to crime in Italy: an analysis of fear of crime and concern about crime as a social problem". Journal of Community \& Applied Social Psychology 15, n. ${ }^{\circ}$ 1: 17-28.

Arslanián, León Carlos. 1998. "Violencia, seguridad ciudadana y orden democrático". Revista Instituto Interamericano de Derechos Humanos-IIDH, $\mathrm{n}^{\circ}$ 28: 69-91.

Braakmann, Nils. 2012. "How do individuals deal with victimization and victimization risk? Longitudinal evidence from Mexico". Journal of Economic Behavior \& Organization 1, n'84: 335-344.

Carbonell, Miguel. 2006. "Cuando la impunidad es la regla. Justicia penal y derechos fundamentales en México". Boletín Mexicano de Derecho Comparado, $\mathrm{n}^{\circ} 6$ : 351-369.

Chadee, Derek, y Nikita Ng Ying. 2013. "Predictors of fear of crime: general fear versus perceived risk". Journal of Applied Social Psychology 9, n43: 1896-1904.

Dammert, Lucía. 2010. "Violencia, crimen e inseguridad en América Latina: desafíos para la democracia”. Academia Superior de Estudios Policiales, nº: 56-87.

Hernández, Luis Hernández. 2008. "México: once retratos de la impunidad". El Cotidiano 150, n²3: 89-100.

Hipp, John. 2010. "Assessing Crime as a Problem: the relationship between Residents' Perception of Crime and Official Crime Rates Over 25 Years". Crime \& Delinquency 4, n59: 616-648.

Matus, Jean Pierre. 1998. "Crisis del derecho e impunidad". Luz et Praxis 2, n4: 17-25.

Navarro, U. 2012. "La seguridad ciudadana y los derechos humanos. Un nuevo paradigma de seguridad en San Luis Potosí". Revista de Derechos Humanos y Estudios Sociales REDHES 4, n. ${ }^{\circ}$ 8: 121-131.

Reyes, Hernán. 2007. "Repensar la inseguridad ciudadana”. Revista Ciudad Segura, México, $\mathrm{n}^{\circ}$ 15: 2-3.

Romero-Salazar, Alexis; Raima Rujano y Miguel Ángel Romero. 2009. "Agresividad cotidiana y aprobación de la violencia extrema”. Revista de investigación científica en Ciencias Sociales, 17(33): 261-280.

Ruiz, José Ignacio. 2007. "Procesos sociales relacionados con el miedo al crimen, la satisfacción con la policía y la victimización: el caso de la cultura ciudadana". International E-Journal of Criminal Science 1, $\mathrm{n}^{\circ} 1$ : 1-29. Acceso el 12 de noviembre de 2014: http://www.ehu. eus/ojs/index.php/inecs/article/view/17

OTROS

Bailey, John y Gustavo Flores-Macías. 2007. "Violent crime and democracy: Mexico in comparative perspective", conferencia presentada en el Midwest Political Science Association's annual meeting, Chicago, IL.

Guerrero, R., et ál. 1994. Disertación sobre la convivencia pacífica (Datos no publicados). Cali, Colombia.

Instituto Nacional de Estadística y Geografía (INEGI). 2005. "Encuesta Nacional Sobre Inseguridad" (ENvipe). México: INEGI.

Instituto Nacional de Estadística y Geografía (inegi). 2011. "Encuesta Nacional de Victimización y Percepción sobre seguridad pública" (enviPe). México: INEGi. Acceso el 6 de junio de 2014.http://www. inegi.org.mx/est/contenidos/proyectos/ encuestas/hogares/regulares/envipe/ envipe2011/default.aspx

Instituto Nacional de Estadística y Geografía (INEGI). 2012. "Encuesta Nacional de Victimización y Percepción sobre seguridad pública" (ENviPe). México: INEGI. Acceso el 6 de junio de 2014.http://www. inegi.org.mx/est/contenidos/proyectos/ encuestas/hogares/regulares/envipe/ envipe2012/default.aspx

Instituto Nacional de Estadística y Geografía (Inegi). 2013. "Encuesta Nacional de Victimización y Percepción sobre seguridad pública" (ENviPe). México: INEGI. Acceso el 6 de junio de 2014.http://www. inegi.org.mx/est/contenidos/proyectos/ encuestas/hogares/regulares/envipe/ envipe2013/default.aspx 
Instituto Nacional de Estadística y Geografía (InEgI). 2014. "Encuesta Nacional de Victimización y Percepción sobre seguridad pública" (enviPe). México: INEGI. ACCESO EL 6 DE JUNIO DE 2014.http://www. inegi.org.mx/est/contenidos/proyectos/ encuestas/hogares/regulares/envipe/ envipe2014/default.aspx

Jusidman, C. 2010. "Introducción". Comisión Nacional para Prevenir y Erradicar la Violencia contra las Mujeres, compilado por M. Cabrera y S. S. García: 5-18. Orígenes de la Violencia en México. Memorias del Foro Interdisciplinario. México: Iniciativa Ciudadana $y$ Desarrollo Social-Incide Social, Ac.

Mier Garza, R. 2010. "Violencia, modernidad y cultura: las expresiones culturales de la devastación social", compilado por Cabrera y S. S. García: 93-116. Orígenes de la violencia en México. Memorias del Foro Interdisciplinario. México: Iniciativa Ciudadana y Desarrollo Social-IIIDE social, AC.

Municipio de Cuernavaca. Plan Integral de Actuación Local para Prevenir la Violencia y la Inseguridad: Municipio de Cuernavaca, Morelos 2009-2012.

Organización Panamericana para la Salud (OPS). 2002. Informe Mundial sobre la violencia y la salud. Organización Mundial de la Salud, Washington DC.

Peace Brigades International-pвi. 2013. Examen periódico universal 2013, Briefing sobre la situación de las personas defensoras de derechos humanos. México: рві. Acceso el 21 de junio de 2014. http:// epumexico.files.wordpress.com/2013/07/ briefing-epu_esp-1.pdf
Programa de las Naciones Unidas (onu). 1985. Octavo Congreso de las Naciones Unidas sobre la Prevención del Delito y Tatamiento del Delincuente. La Habana, Cuba. Acceso el 10 de mayo de 2015. http://www.unodc.org/documents/ congress//Previous_Congresses/8th_ Congress_1990/025_ACONF.144.20_ Note_United_Nations_Norms_and_ Guidelines_in_Crime_Prevention_ana_ Criminal_Justice_S.pdf

Programa de las Naciones Unidas (onu). 2000. Report of the United Nations Consultative Group on the Prevention of Crime and Treatment of Offenders. Michigan: Michigan University Press.

Programa de las Naciones Unidas para el Desarrollo (pnud). 2013. "Seguridad Ciudadana con rostro humano: Diagnóstico y Propuesta para América Latina". Informe Regional de Desarrollo Humano 2013-2014.

Programa de las Naciones Unidas para los Asentamientos Humanos (onuнавітат). 2009. Guía para la prevención local. Hacia políticas de cohesión social y seguridad ciudadana. 2009. México: onu-HabiтAT/Universidad Alberto Hurtado. Acceso el 09 de febrero de 2014. http://www.onuhabitat.org/index. php?option $=$ com_docman\&task=cat view\&gid $=65 \&$ Itemid $=73$

Fecha de ingreso: 13/11/2014 Fecha de aprobación: 17/08/2015 